\title{
LAND COVER CHANGE KNOWLEDGE REPRESENTATION USING TEMPORAL LOGIC AND OPERATION RELATIONS
}

CHEN Jun $^{\text {a }}$, WU Hao ${ }^{\text {b,a }}$

${ }^{a}$ National Geomatics Center of China, Beijing 100830, China, chenjun@nsdi.gov.cn

${ }^{\mathrm{b}}$ School of Remote Sensing and Information Engineering,Wuhan University, Wuhan, 430079, China,whgis@yahoo.cn

\section{Commission VII, WG VII/5}

KEY WORDS: Land Cover, Knowledge Representation, Temporal Logic, Operation Relations, Web Service

\section{ABSTRACT:}

The knowledge representation model plays an important role in the dynamic extracting and web service for Land Cover Change. While former researches were mainly focus on the represent of Spatio-temporal data itself, fewer of them attended the relations of Land Cover Information associated with change. This paper focused on the Land Cover Representation Model, and presented a knowledge representation model for LCC using temporal logic and operation relations. Firstly, we analyses the new demanding of change information service for web publishing, secondly, we discuss the temporal logic of snapshots and increments; then we introduce a method to represent the operation relations between snapshots and increments; finally we construct a knowledge representation for Land Cover Change based on the temporal logic and operation relations.

\section{INTRODUCTION}

Land cover refers to the natural properties and characteristics of a variety of material types covering the surface of global. Since the Land Cover Change (LCC) is one of the significant resources for scientific research (Herold et al., 2008; Xian et al., 2009; Chen et al., 2011), many institutions have built their own land cover websites to fulfill the needs of researchers and public (e.g. USGS, UMD et.al).However, former websites are mainly focused on static information, for example the accessing service, index service, and functional service, which are isolated and incompatible. While the distributed invocations of LCC service require not only static information, but also to provide change services and assemble the services dynamically, which need us to establish knowledge representation model of the Land Cover Change for the dynamic extracting and web service for Land Cover Change.

Despite the technical advances towards spatial-temporal data model, issues related to the knowledge representation of it only recently have been to draw attention (Song et al., 2009; Batsakis et al., 2010). But fewer approaches take, directly or indirectly, the relations of land cover ingredients into account (Turner et al., 1995; Nunes, 1999; Lambin et al, 2003). Indeed the temporal and operation relations among LCC (snapshots and increments) and the computation relations between land cover change and static information are the main points for constructing LCCSP, which ensures the dynamic computation and accessing of implicit information. In previous work, Welty et.al (2006) added temporal relations to TimeSlice and TimeInterval for the representation of temporal objects, which may support the service composition and dynamic extraction of implicit information, while their approach aimed at incontinuous change information and didn't contain the operation or computation relation between spatial data. Di and Ramapriyan (2010) in George Mason built the computation relation of geo-information and functions, and provided a series of computation models to meet the needs of research institutions. However, their approach was taken with the computation of spatial data into a more general level and didn't take the temporal relation into consider. 
The present paper addresses a more specific problem, the knowledge representation focusing on snapshots and increments using temporal logic and operation relations. LCC information poses a knowledge representation problem when extracting implicit information dynamically. To the authors' knowledge, the temporal and operation relations are inherent among snapshots and increments. For example, we can get a snapshot of land cover by a snapshot in other time overlapped with some increments, or get increments by multi-snapshot using the erase operation.

The remainder of the paper is organized as follows. Section 2 discusses the temporal logic among snapshots and increments using matrix arithmetic. Section 3 introduces set operation algorithms into the spatial operations of LCC, and analyses the basic 5 operation relations among snapshots and increments, while section 4 describes the knowledge representation for LCC based on the proposed methodology.

\section{TEMPORAL LOGIC AMONG SNAPSHOTS AND INCREMENTS}

Temporal logic refers to the logical relations among temporal objects. According to the provisions of ISO19100 (2002), temporal object can be divided into "time slice" and "time interval", while "time slice" represents one point in time space, and "time interval" denotes a line with start point, end point and length attributes in time space. Presently, the most popular temporal relations are the 13 relations proposed by Allen (1983) which are shown in Table 1.

\begin{tabular}{|c|c|c|c|}
\hline Relation & Graph & & Inverse Relation \\
\hline Before $(i, j)$ & $\stackrel{\mathrm{i}}{\longleftrightarrow}$ & 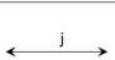 & After $(j, i)$ \\
\hline Meets $(i, j)$ & $\stackrel{i}{\longleftrightarrow}$ & & $\operatorname{MetBy}(j, i)$ \\
\hline Overlaps $(i, j)$ & $\stackrel{i}{\longleftrightarrow}$ & $\stackrel{\mathrm{j}}{\longrightarrow}$ & OverlappedBy $(j, i)$ \\
\hline During $(i, j)$ & $\stackrel{i}{\longleftrightarrow}$ & $\stackrel{j}{\longrightarrow}$ & Contains $(j, i)$ \\
\hline Finishes(i, j) & $\leftarrow$ i & & FinishedBy $(j, i)$ \\
\hline Starts $(i, j)$ & $\longleftrightarrow$ i & $\underset{j}{\mathrm{j}}$ & StartedBy $(j, i)$ \\
\hline Equals $(i, j)$ & $\longleftarrow$ i & $\rightarrow$ & \\
\hline
\end{tabular}

Table 1 Allen's 13 temporal relations
But they are mainly for the relational expression among time intervals. However, LCC information is recorded and expressed primarily through a series of snapshots or increments; while a series of snapshots record the change of a set of discrete "time slice" and increments convey the change within a certain "time interval". Therefore, in the expression of temporal logic for LCC, the temporal relations between "time slice" and "time slice", "time slice" and "time interval", "time interval" and "time interval" must be considered.

In this paper, the snapshot of Land Cover is represented as $S(t)$ and the increment of Land Cover is represented as $I\left(t_{a}, t_{b}\right),(a<b)$. For two snapshots $\mathrm{S}_{1}\left(\mathrm{t}_{1}\right), \mathrm{S}_{2}\left(\mathrm{t}_{2}\right)$, their temporal relations have 3 cases as follows: $t_{1}>t_{2}, t_{1}=t_{2}$ and $t_{1}<t_{2}$. Hence, the temporal relation between $S_{1}$ and $S_{2}$ can be defined as $R\left(S_{1}, S_{2}\right)=$ BBefore, Equals, After $\}$ which can be abbreviated as $R\left(S_{1}, S_{2}\right)=\left\{A_{1}, A_{2}, A_{3}\right\}$. The temporal relation between $S_{1}\left(t_{1}\right)$ and $I_{1}\left(t_{2}, t_{3}\right)$ can be obtained through comparing $t_{1}$ with $t_{2}$ and $t_{3}$ respectively. Therefore the temporal relation of $S_{1}\left(t_{1}\right)$ and $I_{1}\left(t_{2}, t_{3}\right)$, which could be represented as $\mathrm{R}\left(\mathrm{S}_{1}, \mathrm{I}_{1}\right)=\mathrm{R}\left(\mathrm{S}_{1}, \mathrm{~S}_{2}\right) \times \mathrm{R}\left(\mathrm{S}_{1}, \mathrm{~S}_{3}\right)$, can also be shown as below when the relational matrices is adopted to the temporal relations :

$R\left(S_{1}, I_{1}\right)=\left(\begin{array}{l}A_{1} \\ A_{2} \\ A_{3}\end{array}\right)\left(\begin{array}{l}A_{1} \\ A_{2} \\ A_{3}\end{array}\right)=\left(\begin{array}{lll}A_{1} A_{1} & A_{1} A_{2} & A_{1} A_{3} \\ A_{2} A_{1} & A_{2} A_{2} & A_{2} A_{3} \\ A_{3} A_{1} & A_{3} A_{2} & A_{3} A_{3}\end{array}\right)$

Because we have defined $t_{2}<t_{3}$, so in matrices (1) the $A_{i} A_{j}$ does not exist when $\mathrm{i}<\mathrm{j}$ and $=\mathrm{j}=2$, which can be marked as $\varnothing$. Therefore, the formula (1) can be simplified as follow:

$$
\mathrm{R}\left(\mathrm{S}_{1}, \mathrm{I}_{1}\right)=\left(\begin{array}{l}
\mathrm{A}_{1} \\
\mathrm{~A}_{2} \\
\mathrm{~A}_{3}
\end{array}\right)\left(\begin{array}{l}
\mathrm{A}_{1} \\
\mathrm{~A}_{2} \\
\mathrm{~A}_{3}
\end{array}\right)=\left(\begin{array}{ccc}
\mathrm{A}_{1} \mathrm{~A}_{1} & \emptyset & \emptyset \\
\mathrm{A}_{2} \mathrm{~A}_{1} & \varnothing & \varnothing \\
\mathrm{A}_{3} \mathrm{~A}_{1} & \mathrm{~A}_{3} \mathrm{~A}_{2} & \mathrm{~A}_{3} \mathrm{~A}_{3}
\end{array}\right)
$$

Referencing to Allen's 13 kinds of temporal relations, we could obtain that $A_{1} A_{1}=$ before, $A_{2} A_{1}=$ starts, $A_{3} A_{1}=$ during, $A_{3} A_{2}=$ finishes, $A_{3} A_{3}=$ after . Thus, the temporal relation between snapshot and increment is expressed as $\mathrm{R}\left(\mathrm{S}_{1}, \mathrm{I}_{1}\right)=\{$ Before, Starts, During, Finishes, After $\}$, which can be abbreviated as $R\left(S_{1}, I_{1}\right)=\left\{B_{1}, B_{2}, B_{3}, B_{4}, B_{5}\right\}$. Similarly, the temporal relation $I_{1}\left(t_{2}, t_{3}\right)$ and $I_{2}\left(t_{3}, t_{4}\right)$ can be obtained through comparing $\mathrm{S}_{1}\left(\mathrm{t}_{1}\right)$ with $\mathrm{S}_{2}\left(\mathrm{t}_{2}\right)$ and 
$I_{2}\left(t_{3}, t_{4}\right)$ respectively. Therefore the $R\left(I_{1}, I_{2}\right)$ can be shown as $\mathrm{R}\left(\mathrm{I}_{1}, \mathrm{I}_{2}\right)=\mathrm{R}\left(\mathrm{S}_{1}, \mathrm{I}_{2}\right) \times \mathrm{R}\left(\mathrm{S}_{2}, \mathrm{I}_{2}\right)$, which could be represented as follow :

$\mathrm{R}\left(\mathrm{I}_{1}, \mathrm{I}_{2}\right)=\left(\begin{array}{l}\mathrm{B}_{1} \\ \mathrm{~B}_{2} \\ \mathrm{~B}_{3} \\ \mathrm{~B}_{4} \\ \mathrm{~B}_{5}\end{array}\right)\left(\begin{array}{l}\mathrm{B}_{1} \\ \mathrm{~B}_{2} \\ \mathrm{~B}_{3} \\ \mathrm{~B}_{4} \\ \mathrm{~B}_{5}\end{array}\right)$

$=\left(\begin{array}{lllll}\mathrm{B}_{1} \mathrm{~B}_{1} & \mathrm{~B}_{1} \mathrm{~B}_{2} & \mathrm{~B}_{1} \mathrm{~B}_{3} & \mathrm{~B}_{1} \mathrm{~B}_{4} & \mathrm{~B}_{1} \mathrm{~B}_{5} \\ \mathrm{~B}_{2} \mathrm{~B}_{1} & \mathrm{~B}_{2} \mathrm{~B}_{2} & \mathrm{~B}_{2} \mathrm{~B}_{3} & \mathrm{~B}_{2} \mathrm{~B}_{4} & \mathrm{~B}_{2} \mathrm{~B}_{5} \\ \mathrm{~B}_{3} \mathrm{~B}_{1} & \mathrm{~B}_{3} \mathrm{~B}_{2} & \mathrm{~B}_{3} \mathrm{~B}_{3} & \mathrm{~B}_{3} \mathrm{~B}_{4} & \mathrm{~B}_{3} \mathrm{~B}_{5} \\ \mathrm{~B}_{4} \mathrm{~B}_{1} & \mathrm{~B}_{4} \mathrm{~B}_{2} & \mathrm{~B}_{4} \mathrm{~B}_{3} & \mathrm{~B}_{4} \mathrm{~B}_{4} & \mathrm{~B}_{4} \mathrm{~B}_{5} \\ \mathrm{~B}_{5} \mathrm{~B}_{1} & \mathrm{~B}_{5} \mathrm{~B}_{2} & \mathrm{~B}_{5} \mathrm{~B}_{3} & \mathrm{~B}_{5} \mathrm{~B}_{4} & \mathrm{~B}_{5} \mathrm{~B}_{5}\end{array}\right)$

When $i>j, i=j=2$ and $i=j=4$, the value of $\mathrm{B}_{\mathrm{i}} \mathrm{B}_{\mathrm{j}}$ is inexistence, which can be marked as $\varnothing$. Hence, the formula (3) can be abbreviated as follow: $\mathrm{R}\left(\mathrm{I}_{1}, \mathrm{I}_{2}\right)=\left(\begin{array}{ccccc}\mathrm{B}_{1} \mathrm{~B}_{1} & \mathrm{~B}_{1} \mathrm{~B}_{2} & \mathrm{~B}_{1} \mathrm{~B}_{3} & \mathrm{~B}_{1} \mathrm{~B}_{4} & \mathrm{~B}_{1} \mathrm{~B}_{5} \\ \varnothing & \emptyset & \mathrm{B}_{2} \mathrm{~B}_{3} & \mathrm{~B}_{2} \mathrm{~B}_{4} & \mathrm{~B}_{2} \mathrm{~B}_{5} \\ \varnothing & \emptyset & \mathrm{B}_{3} \mathrm{~B}_{3} & \mathrm{~B}_{3} \mathrm{~B}_{4} & \mathrm{~B}_{3} \mathrm{~B}_{5} \\ \varnothing & \emptyset & \emptyset & \emptyset & \mathrm{B}_{4} \mathrm{~B}_{5} \\ \varnothing & \varnothing & \varnothing & \varnothing & \mathrm{B}_{5} \mathrm{~B}_{5}\end{array}\right)$

According to Allen's 13 kinds of temporal relations, we could also acquire

$\mathrm{B}_{1} \mathrm{~B}_{1}=$ before, $\mathrm{B}_{1} \mathrm{~B}_{2}=$ meets, $\mathrm{B}_{1} \mathrm{~B}_{3}=$ overlaps, $\mathrm{B}_{1} \mathrm{~B}_{4}=$ finishedBy, $\mathrm{B}_{1} \mathrm{~B}_{5}=$ contains, $\mathrm{B}_{2} \mathrm{~B}_{3}=$ starts, $\mathrm{B}_{2} \mathrm{~B}_{4}=$ equals , $\mathrm{B}_{2} \mathrm{~B}_{5}=$ startedBy,$\quad \mathrm{B}_{3} \mathrm{~B}_{3}=$ duaring,$\quad \mathrm{B}_{3} \mathrm{~B}_{4}=$ finishes, $B_{3} B_{5}=$ overlapped, $B_{4} B_{5}=$ metBy and $\mathrm{B}_{5} \mathrm{~B}_{5}=$ after. Consequently the temporal relation among increments can be written as $\mathrm{R}\left(\mathrm{I}_{1}, \mathrm{I}_{2}\right)=$ \{Before, Meets, Overlaps, FinishedBy,

Contains, Starts, Equals, StartedBy, During, Finishes, Overlapped, MetBy, After\}, which can be simplified as $\mathrm{R}\left(\mathrm{I}_{1}, \mathrm{I}_{2}\right)=\left\{\mathrm{C}_{1}, \mathrm{C}_{2}, \mathrm{C}_{3}, \mathrm{C}_{4}, \mathrm{C}_{5}, \mathrm{C}_{6}, \mathrm{C}_{7}, \mathrm{C}_{8}, \mathrm{C}_{9}, \mathrm{C}_{10}, \mathrm{C}_{11}, \mathrm{C}_{12}, \mathrm{C}_{13}\right\}$.

While because the change of Land Cover information is continuous, and we can't divide the change into a minim time element, so the snapshots participate in dynamic computation must be started or finished by the increment. So we can add rule 1 to formula (2) and get the simplified relations.

Rule 1: Every $A_{i} A_{j}$ in formula (2), when $i=2$ or $\mathrm{j}=2$, the value of it is $\varnothing$.

So the $R\left(S_{1}, I_{1}\right)$ can be further simplified as follows:

$$
\mathrm{R}\left(\mathrm{S}_{1}, \mathrm{I}_{1}\right)=\left(\begin{array}{ccc}
\varnothing & \varnothing & \emptyset \\
\mathrm{A}_{2} \mathrm{~A}_{1} & \varnothing & \emptyset \\
\varnothing & \mathrm{A}_{3} \mathrm{~A}_{2} & \emptyset
\end{array}\right)
$$

That is to say, the $R\left(S_{1}, I_{1}\right)$ can be written as $\mathrm{R}\left(\mathrm{S}_{1}, \mathrm{I}_{1}\right)=\{$ Starts, Finishes $\}$. When we substitute formula (5) into formula (4), we can also get the simplified temporal relation of $\mathrm{R}\left(\mathrm{I}_{1}, \mathrm{I}_{2}\right)$ as follow:

$$
\mathrm{R}\left(\mathrm{I}_{1}, \mathrm{I}_{2}\right)=\left(\begin{array}{ccccc}
\varnothing & \mathrm{B}_{1} \mathrm{~B}_{2} & \varnothing & \mathrm{B}_{1} \mathrm{~B}_{4} & \varnothing \\
\varnothing & \varnothing & \mathrm{B}_{2} \mathrm{~B}_{3} & \mathrm{~B}_{2} \mathrm{~B}_{4} & \mathrm{~B}_{2} \mathrm{~B}_{5} \\
\varnothing & \varnothing & \varnothing & \mathrm{B}_{3} \mathrm{~B}_{4} & \varnothing \\
\varnothing & \varnothing & \varnothing & \emptyset & \mathrm{B}_{4} \mathrm{~B}_{5} \\
\varnothing & \varnothing & \varnothing & \varnothing & \varnothing
\end{array}\right)
$$

Similarly,

$$
\begin{gathered}
\mathrm{R}\left(\mathrm{I}_{1}, \mathrm{I}_{2}\right)=\{\text { Meets, FinishedBy, Starts, Equals, } \\
\text { StartedBy, Finishes, MetBy }\}
\end{gathered}
$$

In summary, the temporal logic of LCC information can be expressed as follows:

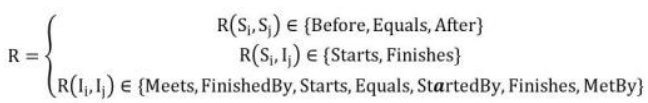

In this formula, $\mathrm{R}\left(\mathrm{S}_{\mathrm{i}}, \mathrm{S}_{\mathrm{j}}\right)$ denotes temporal relations between snapshots, $R\left(S_{i}, I_{j}\right)$ represents temporal relations between snapshot and increment and $R\left(I_{i}, I_{j}\right)$ indicates temporal relations between increments.

\section{THE OPERATION RELATIONS OF CHANGE INFORMATION}

In the previous section, the temporal logic of LCC information is mainly analyzed. But, besides temporal relations there are also spatial operation relationships between snapshots and increments. For instance, we can get the change of Land Cover by the comparing of tow snapshots of the same area, and we can also obtain a newly snapshot by adding some increments to an intrinsic snapshot. Therefore, the spatial operation relations that may exist between snapshots and increments in the expression of change information knowledge should be described. Figure 2 presents LCC information of an area in Beijing, where Figure 2-(a) denotes the snapshot of grass cover in 2000, Figure 2-(b) shows the snapshot data of grass cover in 2010 and Figure 2-(c) represents the reduced grass data from 2000 to 2010. If 2-(c) did not exist in the database, it could be obtained through the operation of 2-(a) and 2-(b).

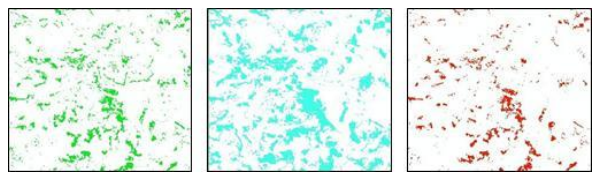

Fig 2. Change Information of Land Cover 2-(a) shows the snapshot of grass cover in 2000 2-(b) shows the snapshot of grass cover in $2010 \quad$ 2-(c) shows the decreased grass from 2000 to 2010 
In the view of the LCC types, we can conclude that the category information of land cover resolves the spatial operation relations between change information. In Figure 2, if land cover types of 2-a and 2-b are both "grass", they might have the "erase" and "erased" relations, and we could obtain the "increased" and "decreased" grass from 2000 to 2010 in this area. If land cover type of 2-b is "forest", there is "intersection" relation between them and we could acquire the land cover that changed from "grass" to "woodland" from 2000 to 2010. Here, from the viewpoint of set operation, we can seem the land cover in an area as a closed set space. Therefore, we may introduce set operation algorithms into the spatial operations of LCC. According to the dynamically generated fashion of LCC, we select set operators of LCC as follows: $\operatorname{erase}(\backslash), \operatorname{erased}(/)$,intersection( $\cap$ ), union( $U$ ) and symmetric difference $(\nabla)$. By this way, any LCC information can be represented as the operation results obtained from these set operators. For example, if $\mathrm{C}$ denotes land cover category, $G$ represents graphics set of land cover in an area and $\mathrm{T}$ indicates time, then the graphics set of snapshots can be expressed as $\mathrm{G}=\operatorname{GeoS}(\mathrm{C}, \mathrm{T})$ and graphics set of increment can be represented as $\mathrm{G}=\operatorname{GeoI}\left(\left(\mathrm{C}_{\mathrm{i}}, \mathrm{T}_{1}\right),\left(\mathrm{C}_{\mathrm{j}}, \mathrm{T}_{2}\right)\right)$, where $\mathrm{T}_{1}$ denotes start time, $T_{2}$ signifies stop time, $C_{i}$ and $C_{j}$ represents land cover category. Suppose $\operatorname{GeoS}\left(\mathrm{C}_{1}, \mathrm{~T}_{1}\right)$ is set $A, \operatorname{GeoS}\left(C_{1}, T_{2}\right)$ is set $B$ and $\operatorname{GeoS}\left(C_{2}, T_{2}\right)$ is set $\mathrm{C}$, four basic spatial operation algorithm as follows can be obtained through the set algorithm:

$$
\begin{aligned}
& \Delta_{+} \operatorname{GeoI}\left(\left(\mathrm{C}_{1}, \mathrm{t}_{1}\right),\left(\mathrm{C}_{1}, \mathrm{t}_{2}\right)\right)=\mathrm{B}-\mathrm{A} \\
& \Delta_{-} \operatorname{GeoI}\left(\left(\mathrm{C}_{1}, \mathrm{t}_{1}\right),\left(\mathrm{C}_{1}, \mathrm{t}_{2}\right)\right)=\mathrm{A}-\mathrm{B} \\
& \Delta_{\cap} \operatorname{Geol}\left(\left(\mathrm{C}_{1}, \mathrm{t}_{1}\right),\left(\mathrm{C}_{2}, \mathrm{t}_{2}\right)\right)=\mathrm{A} \cap \mathrm{C} \\
& \Delta_{\nabla} \operatorname{GeoI}\left(\left(\mathrm{C}_{1}, \mathrm{t}_{1}\right),\left(\mathrm{C}_{1}, \mathrm{t}_{2}\right)\right)=\mathrm{A} \nabla \mathrm{B}
\end{aligned}
$$

And we can also acquire $B$ by the combinatorial operations of formula (1) and (2), which is show as follow:

$$
\begin{aligned}
& \text { B } \\
& =\left(\mathrm{A} \cup \Delta_{+} \operatorname{GeoI}\left(\left(\mathrm{C}_{1}, \mathrm{t}_{1}\right),\left(\mathrm{C}_{1}, \mathrm{t}_{2}\right)\right)\right) \\
& -\Delta_{-} \operatorname{Geol}\left(\left(\mathrm{C}_{1}, \mathrm{t}_{1}\right),\left(\mathrm{C}_{1}, \mathrm{t}_{2}\right)\right)
\end{aligned}
$$

Table 2 shows the semantic description of above five operation relations among snapshots and increments

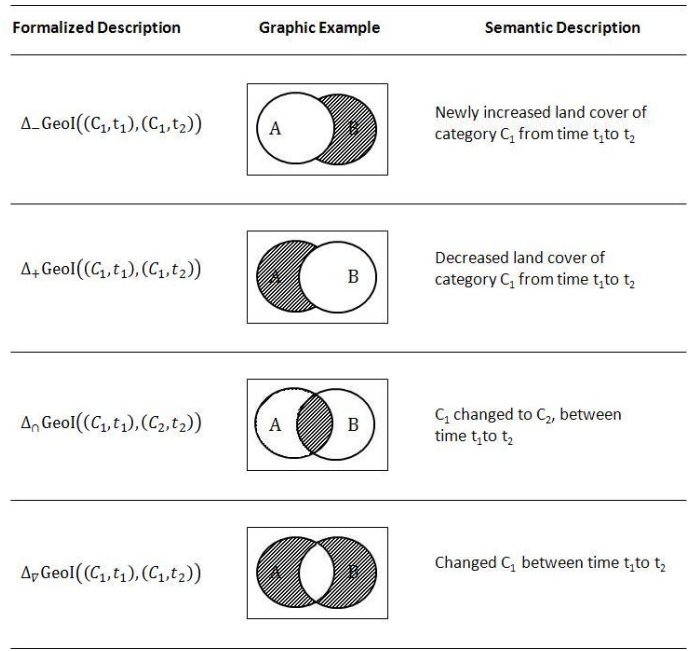

Table 2 The semantic description of operation relation for LCC

In terms of semantic, the above five formulas actually indicate the basic alternative relations between snapshots and increments. The formulas (1), (2), (3) and (4) represent the increments of change information can be acquired by certain spatial operations on the two snapshots. The formula (5) denotes snapshots can be obtained through some spatial operations between snapshots and multi-increments. Based on the combination rules of set operations and further analysis, iterative relationship might exist in these formulas and the snapshot information can be considered as the basic factors for iterative operations.

\section{KNOWLEDGE REPRESENTATION MODEL}

Through the above analyses, it is not difficult to find that graphic elements and land cover category are two fundamental factors of researching and judging LCC information. We can also find that temporal logic and spatial operation relations between sequential snapshots and increments are of great significance to knowledge representation and dynamic extraction for change information. By the conclusion of above research results, we can define the main composition of LCC and relations between them, and get the knowledge representation model of LCC (LCCRM) which is shown in Figure 3. 


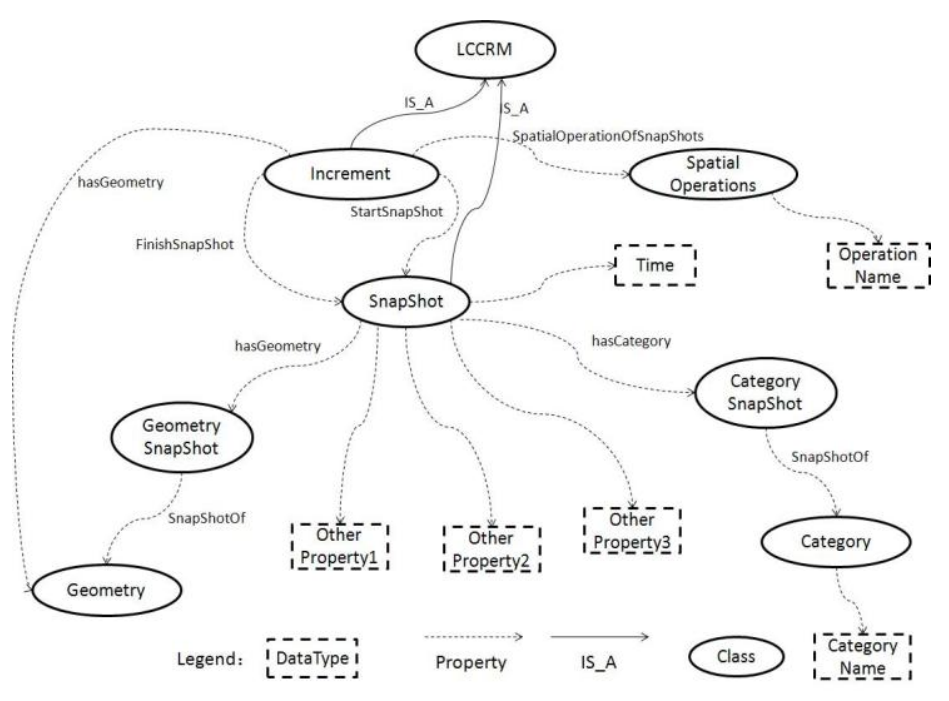

Fig 3. Representation Model of Land Cover Change

The model contains two main subclasses: SnapShot and Increment, while SnapShot includes hasGeometry and hasCategory property, which separately link GeometrySnapShot class and CategorySnapShot class. Besides, SnapShot can aslo contain other static properties. Through SnapShotOf property, GeometrySnapShot and CategorySnapShot can link Geometry class and Category class respectively. Increment class is defined by start and finish SnapShot separately, which are marked as StartSnapShot and FinishSnapShot properties. Increment has independent Geometry property. Furthermore, Increment class aslo stores spatial operation relationships between start and finish SnapShot. The Spatial Operations are marked as Increment's SpatialOperationOfSnapShots property, which is defined by SpatialOperations class. SpatialOperations class includes OperationName property specifically including erase, erased, intersection, union and symmetric difference etc

\section{CONCLULSIONS}

According to temporal and operation relations and expression rules built by the model, sequential snapshots and increments of any time can be described using RDF/OWL language, which contributes to knowledge sharing and reuse of change information and can be used as research foundation of change information service. Its feasibility can be analyzed through three aspects. First, the concept of
LCC information expression includes graphic, category and time and the concept is able to depict change component. Second, the model can describe the temporal and operation relationships between sequential snapshots and increments, and it supports the inference and calculation upon temporal logic and spatial operation. The last aspect is that, all concepts and relations can be displayed through class diagram and support formalized description based on binary relations, which is suitable for current ontology describing rules and auxiliary tools.

Future work includes further refinement of the algorithms and construction of Land Cover Change Information Service, to be able to support Dynamic Service Computing and application system development.

\section{REFERENCES}

Allen J.F., 1983. Maintaining Knowledge about Temporal Intervals. Communications of the ACM26, pp.832-843.

Batsakis S., Petrakis E.G.M., 2010, SOWL: Spatio-temporal Representation, Reasoning and Querying over the Semantic Web. 6th International Conference on Semantic Systems, Graz, Austria, 1-3 September.

Chen J., Chen J., Gong P., Liao A.P., He C.Y., 2011. Higher resolution Global Land Cover Mapping. Geomatics World 02, pp.12-14.

Di L.P., Ramapriyan H.K., 2010. Standards-Based 
ISPRS Annals of the Photogrammetry, Remote Sensing and Spatial Information Sciences, Volume I-7, 2012 XXII ISPRS Congress, 25 August - 01 September 2012, Melbourne, Australia

Data and Information Systems for Earth

Observations - An Introduction. Lecture Notes in

Geoinformation and Cartography.

Foley J., Ruth D., Asner G.P., Barford C., Bonan G.,

Carpenter S.R., Chapin F.S., Coe M.T., Daily G.C., Gibbs H.K., Helkowski J.H., Holloway T., Howard E.A., Kucharik C.J. , Monfreda C., Patz J.A. , Prentice I.C., Ramankutty N., Snyder P.K., 2005. Global consequences of land use, Science, 309:570-574.

Herold M., Mayaux P., Woodcock C.E., Baccini A., Schmulliu C., 2008. Some challenges in global land cover mapping: An assessment of agreement and accuracy in existing $1 \mathrm{~km}$ datasets. Remote Sensing of Environment, 112(5), pp.2538-2556.

ISO / FDIS 19108: 2002 (E) Geographic Information - Temporal schema

LambinE. F., GeistH. J., LepersE., 2003. Dynamics of Land-Use and Land-Cover Change in Tropical regions. Annual Review of Environment and Resources 28, pp.205-241.

Nunes C., 1999. Land-Use and Land-Cover Change (LUCC) Implementation Strategy. The International Geosphere-Biosphere Programme (IGBP).

Song J., Zhu Y.Q., Wang J.L., et al, 2009. A Study on the Model of Spatio-temporal Geo-ontology based on GML. Journal of Geo-Information Science. 11(4), pp.442-451.

Turner B.L., Skole D.L., 1995. Land-Use and Land-Cover Change: Science/Research Plan. The International Geosphere-Biosphere Programme (IGBP).

Welty C., FIKES R., 2006. A Reusable Ontology for Fluents in OWL. Proceeding of the 2006 conference on Formal Ontology in Information Systems: Proceedings of the Fourth International Conference (FOIS 2006), pp.226-236.

Xian G., Homer C., Fry G., 2009. Updating the 2001 National Land Cover Database land cover classification to 2006 by using Landsat imagery change detection methods. Remote Sensing of Environment 113(6), pp.1133-1147. 